\title{
Temporal correlation between oscillating force dipoles drives 3D single cell migration
}

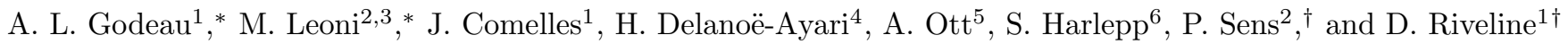 \\ 1 Laboratory of Cell Physics, ISIS/IGBMC, UMR 7104, Inserm, and University of Strasbourg, France \\ 2 Institut Curie, Universit PSL, Sorbonne Universit, CNRS UMR168, \\ Laboratoire Physico Chimie Curie, 75005 Paris, France. \\ 3 Université Paris-Saclay, CNRS, Laboratoire de laccélérateur linéaire, 91898, Orsay, France \\ 4 Univ. Lyon, Université Claude Bernard Lyon 1, \\ CNRS UMR 530610 rue Ada Byron, 69622 Villeurbanne Cedex, France \\ 5 Universität des Saarlandes, Biologische Experimentalphysik, \\ Campus B2.1, D-66123 Saarbrücken, Germany and \\ 6 Tumor Biomechanics, INSERM UMR S1109, Institut d'Hématologie \\ et d'Immunologie 1, place de l'Hôpital, 67200 Strasbourg, France
}

(Dated: March 8, 2021)

\begin{abstract}
Directional cell locomotion requires symmetry breaking between the front and rear of the cell. How this manifests itself for cells moving in physiological 3D matrices is often elusive. We take inspiration from the scallop theorem proposed by Purcell for micro-swimmers in Newtonian fluids: self-propelled objects undergoing persistent motion at low Reynolds number must follow a cycle of shape changes that breaks temporal symmetry. We report similar concepts for cells crawling in 3D. We quantified cell motion using a combination of 3D live cell imaging, visualisation of the matrix displacement and a minimal model with multipolar expansion. We show that cells embedded in 3D matrix form myosin-driven force dipoles at both sides of the nucleus, that locally and periodically pinch the matrix. The existence of a phase shift between the two dipoles is required for directed cell motion which manifests itself in cycles in the dipole-quadrupole diagram, a formal equivalence to the Purcell cycle. We confirm this mechanism by triggering local dipolar contractions with a laser, which leads to directed motion. Our study reveals that the cell controls its motility by synchronising dipolar forces distributed at front and back. This result opens new strategies to externally control cell motion as well as for the design of micro-crawlers.
\end{abstract}

At cellular scales inertia is negligible which puts some particular constraints on self-propelled microscopic objects [1, 2]. Low Reynolds number microswimmers in Newtonian fluids must obey the scallop theorem, stating that the cyclic sequence of shape changes they perform to swim cannot be symmetrical in time [1, 3, 6]. In his seminal work, Purcell illustrated such behaviour as closed trajectories encompassing a finite area in some properly chosen phase space [1. Cells moving in complex environments such as the extracellular matrix are not bound to obey the scallop theorem, which stems from the timereversibility of the Stokes equation. They could instead leverage the visco-elastic nature of the environment [7, 8, or the complex dynamics of adhesion and detachment [9]. This makes the search for unifying principles underlying $3 \mathrm{D}$ cell movement challenging.

To search for generic readouts for cell migration, we designed an assay where cell migration and the spatial distribution of cell matrix interaction could be tracked simultaneously and quantified over time. In our experiment, cells moved in a Cell Derived Matrix (CDM), obtained using a protocol adapted from [10. Briefly, a confluent monolayer of NIH3T3 cells expressing fluorescently labeled fibronectin was triggered to synthesise a thick $(13 \mu \mathrm{m}$ - Fig.S1) matrix. Cells were removed after eight days by lysis. The resulting CDM was physiologi-

\footnotetext{
* These two authors contributed equally

$\dagger$ Corresponding authors: pierre.sens@curie.fr riveline@unistra.fr
}

cal and sufficiently soft and elastic to relate cell dynamics to matrix deformation (Fig 1 and Material and Methods I.B.1), see also [11. The CDM behaved as a soft and elastic material of elastic modulus $\approx 50 \mathrm{~Pa}$ as determined by optical tweezers micromanipulation of embedded beads, see Material and Methods I.B.5, Fig.S1 and Movie 1. For migration experiments, cells were plated at low density, spontaneously penetrated the matrix and could be followed individually. The CDM was easily deformed by cells as they moved (Fig 1 a, b and Movies 2-3-4). This enabled us to quantify the associated matrix deformation [12] via the Kanade-Lucas-Tomasi (KLT) tracker method [13] (Fig[1 b). In our assay we image the network deformation, the cellular machinery responsible for force-generation and the focal contacts which promote adhesion and force transmission between the cell and the CDM. Key molecular players were fluorescently labelled: (i) the fibronectin network within the CDM (Fig 1 12); (ii) components of the cell cytoskeleton, acto-myosin and microtubules (Fig 1 a, c, d and Movies 3,5); and (iii) zyxin in focal contacts (Fig.S2, Fig.S5 and Movie 4).

During observation some wild-type cells showed persistent motion while others did not move. Interestingly both motile and non-motile cells exhibited local zones of contraction-extension, equivalent to two force dipoles on either side of the nucleus (Fig $1 \mathrm{~b}$ ). These deformations were cyclic, with periods of $\approx 10$ mins and amplitudes of typically $2.5 \mu \mathrm{m}$, reminiscent of previous reports of cell autonomous contractile oscillations [14. Periods of oscillations were comparable for motile and non-motile 
bioRxiv preprint doi: https://doi.org/10.1101/2020.05.07.081984; this version posted March 8, 2021. The copyright holder for this preprint (which was not certified by peer review) is the author/funder, who has granted bioRxiv a license to display the preprint in perpetuity. It is made available under aCC-BY-ND 4.0 International license.

cells (Fig]2, g,i), see Material and Methods I.B.6 for quantification of the oscillations. The phases of contraction correlated with the formation of local myosin clusters (Fig 1 c). This is consistent with the observation of distinct myosin-driven contraction centres in the migration of neurons on 2D surfaces [15. We observed more protrusive activity at one of the two ends of elongated cells, in both motile and non-motile cells. This apparent spatial polarisation was not sufficient to elicit directed motion. On the other hand, the temporal cross-correlation function of contraction-extension at the two cell ends revealed striking differences between motile and non-motile cells. While non-migrating cells showed no clear phase shift between the two ends, with a cross-correlation peak at $t=0$, migrating cells showed a persistent time lag of $\approx 2$ min $(\operatorname{Fig} 2 \mathrm{j}, \mathrm{k})$. A system undergoing directed motion must exhibit time reversal asymmetry. In our case, this asymmetry manifests itself through a time lag between the contraction-extension cycles of the two force dipoles.

The most intuitive way to visualise time reversal symmetry breaking is through the existence of cycles in a

a
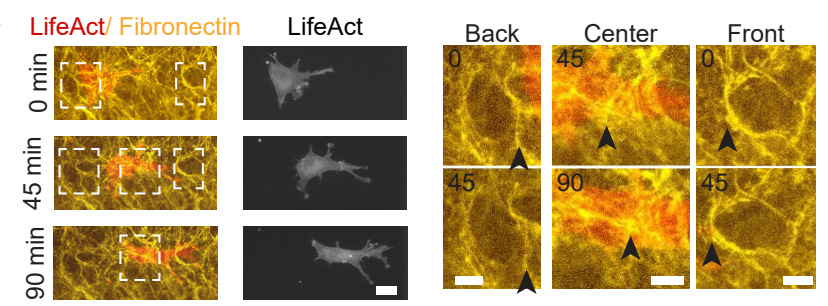

b
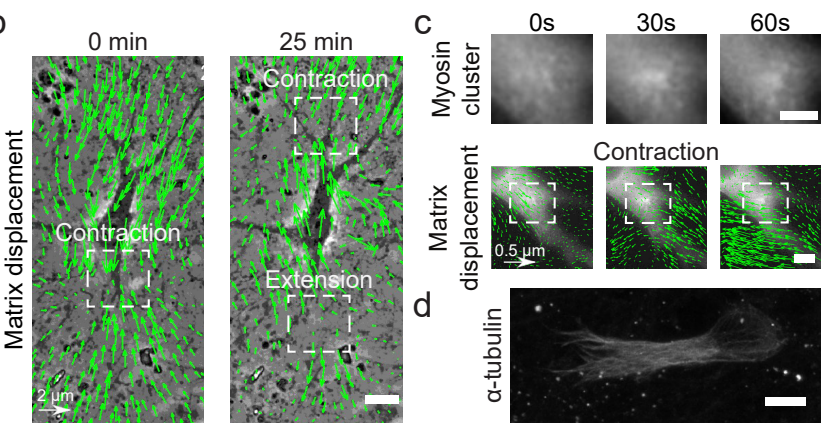

FIG. 1. Key players in cell motility. a) Left panel (and Movie 4): A cell deforms the fibronectin (FN) network when migrating (FN in yellow and mCherry LifeAct for actin filaments in red). Right panel: Enlargement of the white windows of the left panel. Black arrows highlight displacement of fibers due to cell movement. b) Overlay of phase contrast image and KLT calculation of mesh displacement (green arrows - with scale bar shown) with local contraction and extension dipoles indicated with white windows. c) Myosin clusters form locally within cells and are correlated with local contraction. Bottom: KLT deformation (green arrows) and myosin mCherry signal. Top: Enlargement of the white window of the bottom panel showing the myosin mCherry signal. d) $\alpha$-tubulin staining of a cell inside CDM. Microtubules extend from the centrosome to the periphery of the cell (see also Movie 5). Scale bars: (a) $25 \mu \mathrm{m}$ (10 $\mu \mathrm{m}$ in the enlargments) (b,c,d) $10 \mu \mathrm{m}$.
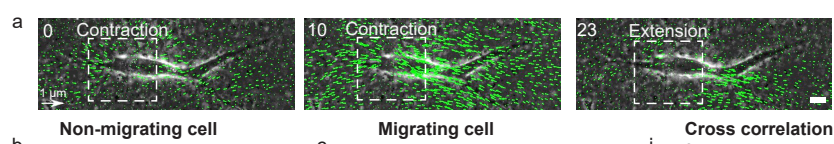

b Direction of polarization Cell axis

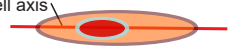

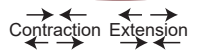
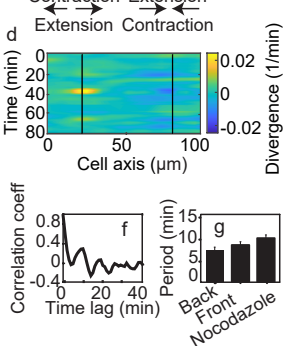

Migrating cell

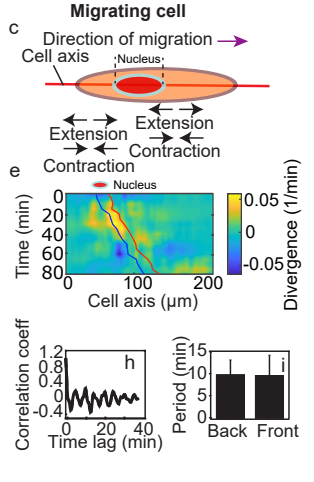

Cross correlation ront-back matrix displacement
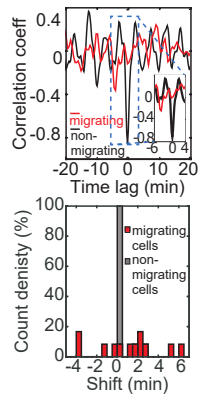

FIG. 2. Dynamics of matrix deformation for migrating and non-migrating cells. a) Snapshots overlaying phase contrast images and KLT calculation of matrix rate of deformation (green arrows) showing a contraction/extension center, scale bar $10 \mu \mathrm{m}$, time in minutes. b-c) Schematics of the alternating phases of contraction and extension for a non-migrating cell (b) and a migrating cell (c). d-e) Heatmap of the divergence of the corresponding matrix displacement. Contractile and extensile force dipoles correspond to blue and yellow spots, respectively. Non-migrating cells (d) show two oscillating dipoles (their centres are approximately indicated by the solid black lines) that appear to be in phase opposition, while migrating cells (e) show a more complex spatio-temporal pattern. The blue and red solid lines in (e) indicate the two sides of the nucleus. f) Typical correlation function of the contraction-extension time series at the back of non-migrating cells, with a first peak at $\approx 5$ min. g) Average periods of contraction-extension cycles, which is similar $(\approx 7-8 \mathrm{~min})$ at the front and back of the cell, and slightly higher $(\approx 9.5 \mathrm{~min})$ for nocodazole treated cells, which show no permanent cell polarisation. h) Correlation function at the back of migrating cells, with a first peak at $\approx 10 \mathrm{~min}$. i) Average period of the contraction-extension cycles for migrating cells. j ) Typical cross-correlation function between back and front rates of deformations. k) Distribution of the values of the time-lag for migrating and non-migrating cells (with $n_{m o t}=12$ motile cells, $n_{\text {nomot }}=8$ non-motile cells, and $N>3$ biological repeats).

properly chosen phase space. This phase space may be based on a multipole expansion of the traction force exerted by the cell on its surrounding. This approach was pioneered by Tanimoto \& Sano for Dictyostelium discoideum crawling on deformable 2D substrate [2]. In particular, they showed that crawling Dictyostelium discoideum exhibits cycles of finite area in the dipolequadrupole phase space. In the present work, the traction forces exerted by the cell are analysed indirectly through the deformation of the matrix. Indeed, our characterisation of the mechanical properties of the CDM show that it locally behaves as a linear elastic material with a well defined elastic modulus (Material and Methods I.B.5, Fig.S1). This supports the approxima- 
a

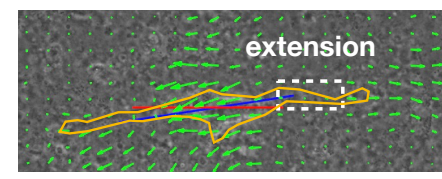

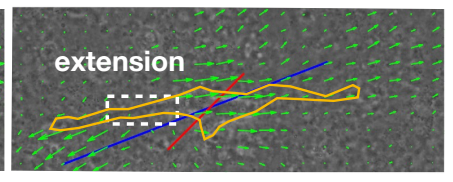

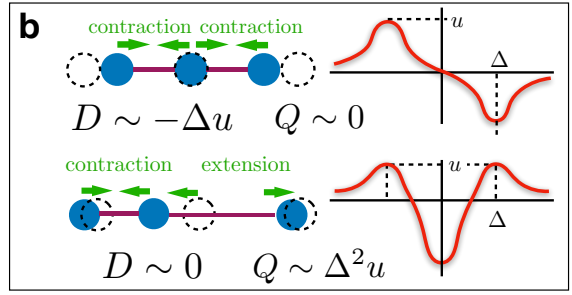

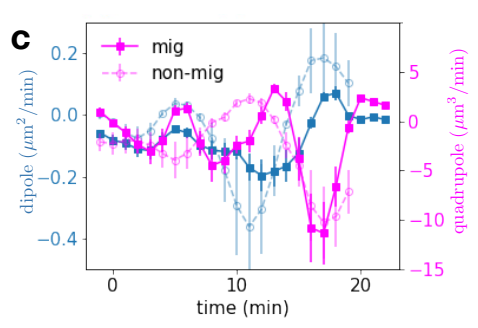

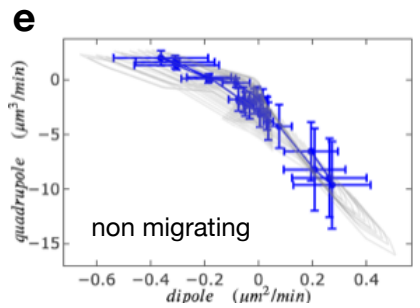

$\mathbf{f}$
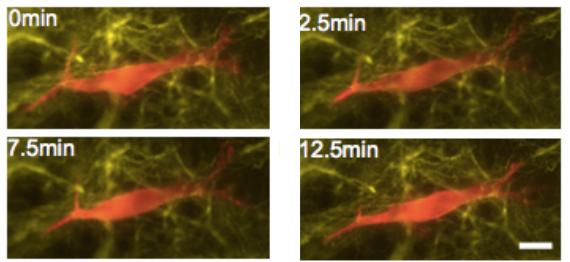

g

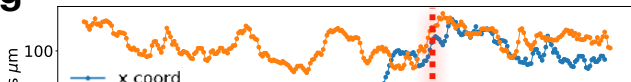

d

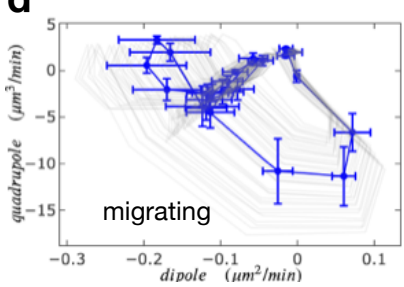

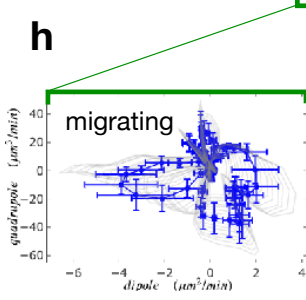

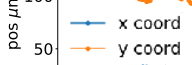

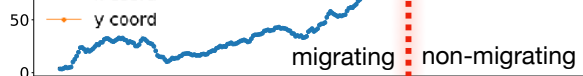

FIG. 3. Multipole analysis of the matrix deformation rate. a) Snapshots of a cell with: matrix rate of deformation, green arrows, the main dipole axis, blue, the axis of the cell motion, red. b) Schematic representation of dipoles $(D)$ and quadrupoles $(Q)$ of the 1D matrix displacement (rate) distributions. The distribution on top has non-zero dipole but vanishing quadrupole, and that on the bottom has vanishing dipole and non-zero quadrupole. c) Time series of the main dipole, blue, and quadrupole, magenta, - projected on the cell axis - for a migrating cell (squares) and a non-migrating cell (circles), sampling approximatively $1 / 10$ of the duration of the entire experiment. d-e) Cell trajectory in the dipole/quadrupole phase space for a migrating cell (d) and a non-migrating cell (e). The migrating cell follows a cycle with a finite area and the non-migrating cell does not. The error bars are obtained following the procedure described in Material and Methods I.B.7. The individual cycles for different radii are shown in light gray. f) Snapshots of a cell which in the course of the same experiment displays a transition from migrating to non-migrating behaviour (Lifeact in red and fibronectin in yellow), scale bar $10 \mu \mathrm{m}$. (g) Cell positions in the $\mathrm{x}-\mathrm{y}$ plane (blue and orange curves) showing a transition from migrating to non-migrating phase (red dashed line at around 32min). (h) Trajectories in the dipole/quadrupole phase space for three different time intervalles showing cycles with finite area in the migrating phase and with vanishing area in the non-migrating phase (see also Movie 7).

tion of a linear relationship between the moments of the force distribution and the moments of the resulting matrix displacement distribution. Note however that the relationship could be more complex due to matrix heterogeneities.

We analysed the $2 \mathrm{D}$ projection of the $3 \mathrm{D}$ deformation field of the CDM and compute the dipolar and quadrupolar moments of the rate of matrix deformation, Material and Methods I.B.4. Briefly (see details in Material and Methods I.B.7), we extracted the 2D projected velocity vector field $u_{i}^{(n)}$ for the component $i$ of the change of substrate deformation between two consecutive frames at position $n$ of the mesh. The dipole is a tensor defined as $S_{i j}=\frac{D_{i j}+D_{j i}}{2}$ with $D_{i j}=\sum_{n} \Delta_{i}^{(n)} u_{j}^{(n)}$, where $\Delta_{i}^{(n)}$ is the i-th component of the vector joining the cell centre and the point $n$ on the mesh. Similarly, the quadrupole tensor is defined as $Q_{i j k}=\sum_{n} \Delta_{i}^{(n)} \Delta_{j}^{(n)} u_{k}^{(n)}$. We then consider the projection of these quantities on the main axis identified by the average direction of the cell trajectory and we call these components the main dipole, D, and the main quadrupole, Q , see Fig $3 \mathrm{p}$ for a sketch of the physical/geometrical meaning of these quantities.
We determine the time variation of the dominant components of the dipole and quadrupole matrices. The dipole/quadrupole observed for non-migrating cells are comparable in magnitude to those of migrating cells (Fig $3 \mathrm{a}, \mathrm{b})$. This shows that the absence of migration is not due to the lack of traction forces (see also Fi.S4). The cell trajectory represented in the dipole/quadrupole phase space showed a cycle enclosing a finite area for migrating cells, but a vanishing area for non-migrating cells (Fig 3 c,d, and more examples Fig.S5). To further test the relationship between finite cycle area and motility of individual cells, we analyzed the migration of the same cell that underwent motion and then stopped (Fig $3 \mathrm{f}-\mathrm{h}$ and Movie 7). Strikingly, we observed that the cycles switched from a finite area while the cell was moving to a vanishing area when the cell stopped (Fig $3 \mathrm{~h})$. The finite area is a direct illustration of the fact that the phase shift between the contraction-extension cycles at the two ends of migrating cells observed in Fig.2 $\mathrm{j}, \mathrm{k}$ also manifests itself in the pattern of matrix displacement. This is a clear signature of time reversal symmetry breaking.

To test whether we could correlate the oscillations at the two cell ends with molecular actors, we tracked cell 
motion in the presence of the microtubule depolymerizing agent, nocodazole. Nocodazole treated cells undergo oscillations driven by local force dipoles with typical periods similar to wild type cells $(\sim 10 \mathrm{~min}$, Fig $2 \mathrm{~g})$ but do not maintain a fixed cell-polarization (Movie 12). In this case, oscillations at different cell ends were in phase and cells did not show directed motion. This suggests that the coupling between oscillators needed to promote directed motion could involve microtubules. Indeed, the microtubular network spans the entire the cell body (Fig.1d and Movie 5). It seems natural to assume that this transfers information between the two sides of the cell. This suggestion is consistent with the notion that the microtubular network regulates the polarity of migrating cells [16, 17.

The geometry and dynamics of the distribution of matrix displacement call for a direct comparison with models of self-propelled objects made of discrete moving beads [3, 4, 6, 8, 9, 19. Fig 44,b displays an idealised cell with two pairs of beads exerting time-shifted oscillatory force dipoles at its two ends. The cell activity is characterised by the amplitude $d$ and period $T$ of the oscillations and a phase shift $\psi=2 \pi \Delta T / T$ between oscillations at the two ends. The simplest self-propelled object is a micro-swimmer embedded in a newtonian fluid and migrating due to hydrodynamic interactions $3,4,6$, In this case, the period of oscillation is the only time scale in the problem and, in the limit of small oscillation amplitudes, the net cell velocity over a cycle follows the scaling [6]

$$
V_{\text {swim }}=\frac{d^{2}}{L_{s} T} f_{s}(\psi)
$$

where $f_{s}(\psi)$ is a periodic function of the phase shift satisfying $f_{s}(\psi=0)=0$, i.e. no velocity without phase shift, as required by the scallop theorem, and $L_{s} \propto r^{4} /\left(a D^{2}\right)$ is a length scale entirely set by the cell geometry $(r$ is the distance between the dipoles, $D$ the dipole size and $a$ the bead size - Fig, 4 a).

A key aspect of cell crawling, which is absent for microswimmers, is the dynamics of cell attachment and detachment from the surrounding matrix. Our observations suggest that dipole contraction is associated with an active contraction of acto-myosin clusters and that dipole extension corresponds to the elastic relaxation of the CDM following local cell detachment, i.e. the loss of focal contacts, see Movie 4 and 20]. The kinetics of cell binding/unbinding to the extracellular matrix defines additional dynamic parameters, which can at the simplest level, be captured by a velocity scale $v_{a d h}$ [19. For cells crawling on a rigid substrate - and in the limit of small oscillation amplitudes and fast binding kinetics - the net crawling velocity can be written as [19]:

$$
V_{\text {crawl }}=\frac{d^{3}}{v_{a d h} L_{c} T^{2}} f_{c}(\psi)
$$

where $f_{c}(\psi=0)=0$ as for swimmers, and the lengthscale $L_{c} \propto r^{2} / a$ also includes additional dimensionless factors related to substrate dissipations (see [19] for more details).

These simple models predict how the velocity should vary with the period of oscillation $T$. The result depends on whether the amplitude of oscillations $d$ is fixed or is a function of $T$. For oscillations of constant amplitude, the net velocity of both swimmers and crawlers decreases if the period of oscillation increases. On the contrary, the velocity is expected to increase with the period if the amplitude increases linearly with the period: $d \sim T$, as can be expected if the self-propelled object operates under constant force - or equivalently constant contraction/extension rate.

a Direction of migration $\longrightarrow$
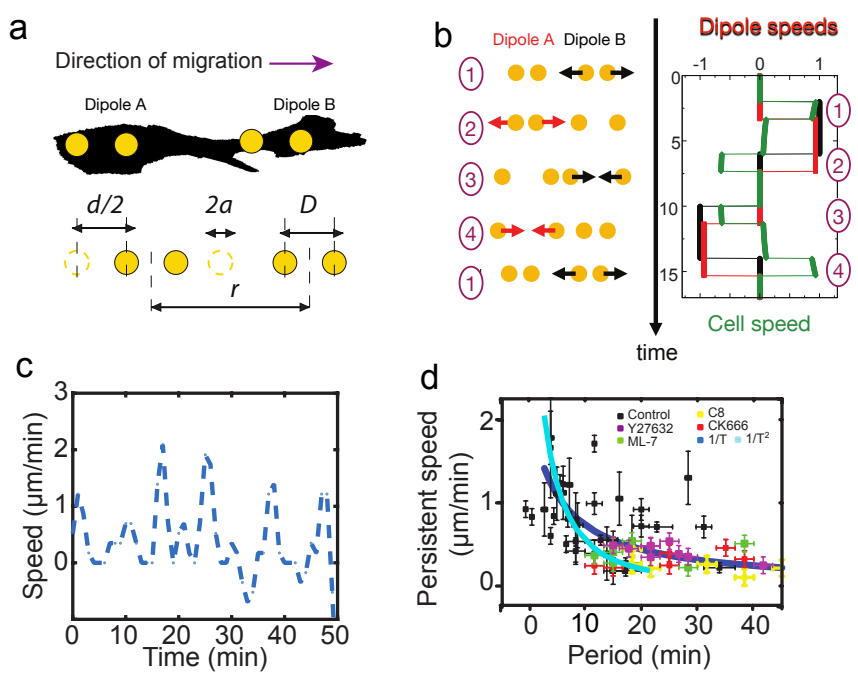

FIG. 4. Persistent speed is related to the period of oscillations. a) Schematics of dipoles distribution highlighting quantities used in the theoretical model: two dipolar units ("A" and "B") made up of disks of radius $a$, through which cells exert traction forces on the extracellular environment. The dipoles, at distance $r$ apart, oscillate with period $T$, with minimum amplitude $D$ and a maximum amplitude $D+d$. b) Model dynamics. Left: Alternate phases of extension/contraction are imposed to the two dipoles, defining a cycle ("1, 2, 3, 4, 1...") that is not time-reversible. Right: the extension/contraction rates of dipole "A" and "B" are shown in red and black, respectively, in unit $d / T$. The cell velocity, calculated using the model discussed in [6], is shown in green in the same units. It oscillates between positive and negative values - with a non-vanishing mean - with a period equal to that of individual dipoles. c) Typical plot of the experimentally measured instantaneous speed of a migrating cell over time, showing oscillation with a non-vanishing mean. d) Persistent speed as a function of speed period for control cells and cells treated with specific cytoskeleton inhibitors: $10 \mu \mathrm{M}$ ROCK inhibitor Y-27632; $10 \mu \mathrm{M}$ MLCK inhibitor ML-7; 100 $\mu \mathrm{M}$ lamellipodia growth promoter $\mathrm{C} 8$ [18] and $50 \mu \mathrm{M}$ Arp2/3 inhibitor CK666, see Material and Methods I.B.2. Each data point corresponds to one cell. The plot displays a decay consistent with a power law. The continuous lines show the fits for $V \sim 1 / T$ (dark blue) and $V \sim 1 / T^{2}$ (light blue), following Eqs. [12]. 
The temporal oscillations of the instantaneous cell velocity is a good readout for the dynamics of internal force generation. For such self-propelled objects, the instantaneous velocity oscillates around the average values given by Eqs 1 or 2 with a time dependence that reflects the dynamics of the underlying force dipoles. An example of such (theoretical) velocity oscillations can be seen on Fig 4 b. Experimental observations indeed report strong oscillations of the instantaneous cell velocity (Fig, $4 \mathrm{c}$ ).

To test how the period of oscillation of migrating cells influenced their velocity, we tracked cell motion for a variety of specific inhibitors that impact on oscillation periods, see Material and Methods I.B.6, Fig.S3 and Movies 10-12. We plotted the cell velocity as a function of oscillation period for all conditions (Fig $4 \mathrm{~d}$ ). An inverse correlation between period and velocity is evident from the data. This is consistent with locomotion being driven by controlling cell deformation instead of cell traction forces. Similar conclusions have been reached in the different context of adherent epithelial cells [21.

Altogether, these results suggest that the temporal coupling between spatially distributed force dipoles along the cell promotes cell motion. To verify this, we needed to find a way to externally trigger localised cellular force dipoles. We leveraged the fact that localized laser ablation led to the recruitment of actin cytoskeleton and localised cell contraction associated to a pinching of the matrix (Fig:5 a-b and Movie 8). We then used this method to locally impose cellular force dipoles, by triggering local contractions alternatively at either sides of the cell. We imposed correlated contractions by selecting a constant time interval between consecutive laser ablations: this triggered translocation. This shows that externally induced force-dipoles are sufficient to promote directed cell motion (Fig 5 c-d and Movie 9).

Acto-myosin complexes are likely to be the functional elements that control the dynamics of the individual contractile units. Remarkably, the dynamics of individual dipoles appear similar in migrating and non-migrating cells, suggesting that the same force generation machinery is equally active in both types of cells, and that it is the synchronisation between individual units that makes movement possible. Altering the dynamics of individual units can affect motion, in particular, faster oscillations can lead to faster motion, but the coordination between units is key in enabling cell translocation.

Acto-myosin networks commonly show oscillatory dynamics in vitro and in vivo in a variety of systems and over a large range of length scales [14: single filaments in motility assays [22 24], cells [25] and cell fragments [26], and even entire organisms 27]. The biological function of these generic dynamics is often unclear. However in our case, they appear to be essential. We conjecture that cellular systems could adapt their velocity by modulating the oscillation period. In this context, it would be interesting to test this proposal by combining tracking of single moving cells and local matrix deformations in vivo. If confirmed, this would provide an outstanding example

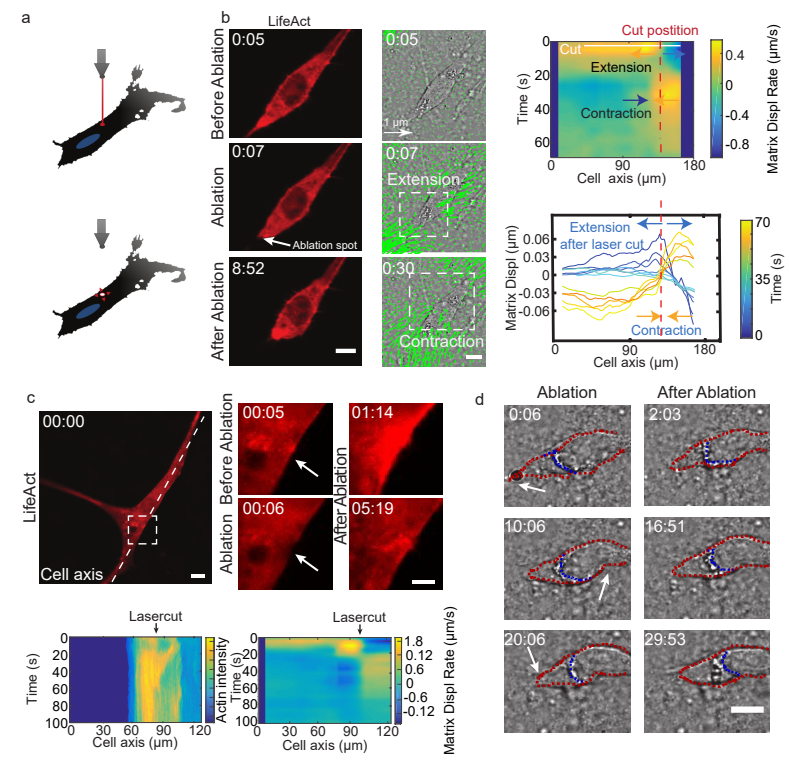

FIG. 5. Cell motion is triggered by means of laser induced force dipoles. a) Schematic of laser ablation experiment. b) (Left panel: Lifeact, middle panel: phase contrast and KLT) Ablation at the back of the cell (white arrow) immediately followed by an extension, and later by a contraction of the matrix, (both highlighted using white square window). scale bar LifeAct: $10 \mu \mathrm{m}$ KLT: $20 \mu \mathrm{m}$. Right panel. Bottom: Plot of the displacement rate along the cell axis at different time (colour coded) showing extension and contraction. Top: Heatmap of displacement rates indicating the initial extension and the subsequent contraction. c) Top: sequence of snapshots during laser ablation on cell expressing mCherry LifeAct. Intensity drops locally immediately after the cut, followed by a local recruitment of actin, scale bar $20 \mu \mathrm{m}$, scale bar in zoom $5 \mu \mathrm{m}$. Bottom: the intensity heatmap reveals a focused actin flow (see also Movie 8). As shown in the deformation map, the contraction precedes this flow. d) Consecutive ablations (indicated with white arrows) mimics contraction-extension cycles at the front and back of cell. Ablation is performed in the following order: at the cell back, at the front and then at the back again. In all panels, scale bar $10 \mu \mathrm{m}$ and time in $\mathrm{mm}: \mathrm{ss}$. Note cell motion to the right (see also Movie 9), scale bar $10 \mu \mathrm{m}$. The cell is outlined in red and the back of the nucleus with a blue dashed line.

of a physiological relevance for such oscillations.

The phase-shift between contractile units encodes cell polarity. Its maintenance in the course of time requires the existence of a polarity memory. If this type of phase locking can be expected in non-linear systems [28, it is more demanding in the cellular context, where it is challenged by strong fluctuations in protein concentrations and activities. Our results suggest that the microtubular network is involved. However, other cytokeletal elements and their interplay with adhesion dynamics are likely to play a role as well. Disentangling the interplay between mechanics and biochemical regulation in this process remains an important open question.

Cells moving in 2D or in micro-channels often display clear spatial polarisation, characterised by F-actin flow- 
ing from the front to the back of the cell - so-called actin retrograde flow $-29,30$. In this case, the lack of time reversal symmetry - the fact that the system looks different when the movie is played backward - clearly manifests itself by the existence of a coherent actin flow at the scale of the entire cell. Consequently, the cell velocity is directly related to the dynamics of the actin flow. Many cells, especially mesenchymal cells moving in 3D matrices, do not show such polarised actin flow, but instead display sequences of protrusion/retraction at both ends of the cell. Although the local actin dynamics within these protrusions is comparable to whole cell retrograde flow in fast moving cells, the existence of multiple competing protrusions leads to frequent stochastic retraction 31. Such cells may nevertheless display directed motion, but at much slower net velocity. It is then challenging to identify the occurence of time reversal symmetry breaking. Our results show that the lack of time reversal symmetry in cells moving in 3D CDM is associated to a time shift between the oscillating dynamics of the two cell ends.

We propose that temporal correlations between distinct contraction-extension units along the cell body is a general principle used by mesenchymal cells to achieve directional motility in 3D. This suggests new strategies to control the motion of cell by externally modulating the local contractile activity, for which we give a proofof-principle using a standard laser setup. This concept could also be used to design synthetic micro-crawlers.
Whereas there exists many examples of artificial microswimmers (see 32 for a review), there is to our knowledge no realisation of micro-crawler in regimes where inertia is negligible.

\section{ACKNOWLEDGMENTS}

We thank the Riveline Lab. for discussions and help, M. Maaloum, the Imaging and Microscopy Platform of IGBMC, and H.P. Erickson, A. Huttenlocher, E. Paluch for constructs, J. Goetz for the former CDM protocol and M. Piel for critical reading of the manuscript. D.R. acknowledges support from CNRS (ATIP), ciFRC Strasbourg, the University of Strasbourg, Labex IGBMC, Foundation Cino del Duca, Région Alsace, Saarland University. A. Ott and D. Riveline acknowledge support from DFH-UFA through the Collège Doctoral FrancoAllemand CDFA-01-13. This study with the reference ANR-10-LABX-0030-INRT has been supported by a French state fund through the Agence Nationale de la Recherche under the frame programme Investissements d'Avenir labelled ANR-10-IDEX-0002-02. M.L. acknowledges financial support from the ICAM Branch Contributions and Labex CelTisPhyBio No ANR-10-LBX-0038 part of the IDEX PSL No ANR-10-IDEX-0001-02 PSL. A. Ott acknowledges support by DFG within the collaborative research center SFB 1027.
[1] E. M. Purcell, American Journal of Physics 45, 3 (1977).

[2] H. Tanimoto and M. Sano, Biophys. J. 106, 16 (2014).

[3] A. Najafi and R. Golestanian, Phys Rev E Stat Nonlin Soft Matter Phys 69, 062901 (2004).

[4] R. Golestanian and A. Ajdari, Phys Rev E 77, 036308 (2008).

[5] N. P. Barry and M. S. Bretscher, Proc Natl Acad Sci USA 107, 11376 (2010).

[6] M. Leoni and P. Sens, Phys. Rev. E 91, 022720 (2015).

[7] T. Qiu, T.-C. Lee, A. G. Mark, K. I. Morozov, R. Münster, O. Mierka, S. Turek, A. M. Leshansky, and P. Fischer, Nature Communications 5, 5119 (2014)

[8] C. Datt, B. Nasouri, and G. J. Elfring, Phys. Rev. Fluids 3, 123301 (2018)

[9] G. L. Wagner and E. Lauga, Journal of Theoretical Biology 324, 42 (2013).

[10] E. Cukierman, R. Pankov, D. R. Stevens, and K. M. Yamada., Science 294, 1708 (2001).

[11] R. J. Petrie, N. Gavara, R. S. Chadwick, and K. M. Yamada, J Cell Biol 197, 439 (2012).

[12] H. Delanoe-Ayari et al., Cytoskeleton 65, 314 (2008).

[13] B. D. Lucas and T. Kanade, International Joint Conference on Artificial Intelligence 2, 674 (1981).

[14] K. Kruse and D. Riveline, Curr Top Dev Biol 95, 67 (2011)

[15] J. Jiang, Z. Zhang, X.Yuan, and M. Poo, J. Cell Biol. 209, 759 (2015).

[16] S. Etienne-Manneville, Traffic 5, 470 (2004)
[17] I. Kaverina and A. Straube, Seminars in cell \& developmental biology 22, 968 (2011)

[18] I. Nedeva, G. Koripelly, D. Caballero, L. Chieze, B. Guichard, B. Romain, E. Pencreach, J.-M. Lehn, M.-F. Carlier, and D. Riveline, Nat Commun. 4, 2165 (2013).

[19] M. Leoni and P. Sens, Phys. Rev. Lett. 118, 228101 (2017).

[20] A. Godeau, H. Delanoë-Ayari, and D. Riveline, Methods Cell Biol. 156, 185 (2020).

[21] A. Saez, A. Buguin, P. Silberzan, and B. Ladoux, Biophysical journal 89, L52 (2005)

[22] D. Riveline, A. Ott, F. Julicher, D. A. Winkelmann, O. Cardoso, J. J. Lacapere, S. Magnusdottir, J. L. Viovy, L. Gorre-Talini, and J. Prost, Eur Biophys J. 27, 403 (1998).

[23] P.-Y. Plaçais, M. Balland, T. Guérin, J.-F. Joanny, and P. Martin, Phys Rev Lett 103, 158102 (2009).

[24] D. Gillo, B. Gilboa, R. Gurka, and A. BernheimGroswasser, Phys. Biol. 6, 036003 (2009).

[25] J. Negrete et al., Phys. Rev. Lett. 117, 148102 (2016).

[26] E. Paluch, M. Piel, J. Prost, M. Bornens, and S. C., Biophys J. 89, 724 (2005).

[27] A. C. Martin, M. Kaschube, and E. F. Wieschaus, Nature 457, 495 (2009).

[28] A. Pikovsky, M. Rosenblum, and J. Kurths, Synchronization: A Universal Concept in Nonlinear Science (Cambridge University Press, 2002). 
[29] L. Barnhart et al., PloSBiology 9, e1001059. (2011).

[30] Y.-J. Liu, M. Le Berre, F. Lautenschlaeger, P. Maiuri, A. Callan-Jones, M. Heuzé, T. Takaki, R. Voituriez, and M. Piel, Cell, Cell 160, 659 (2015)
[31] D. Caballero, R. Voituriez, and D. Riveline, Biophys. J. 107, 34 (2014).

[32] J. Elgeti, R. G. Winkler, and G. Gompper, Reports on Progress in Physics 78, 056601 (2015). 